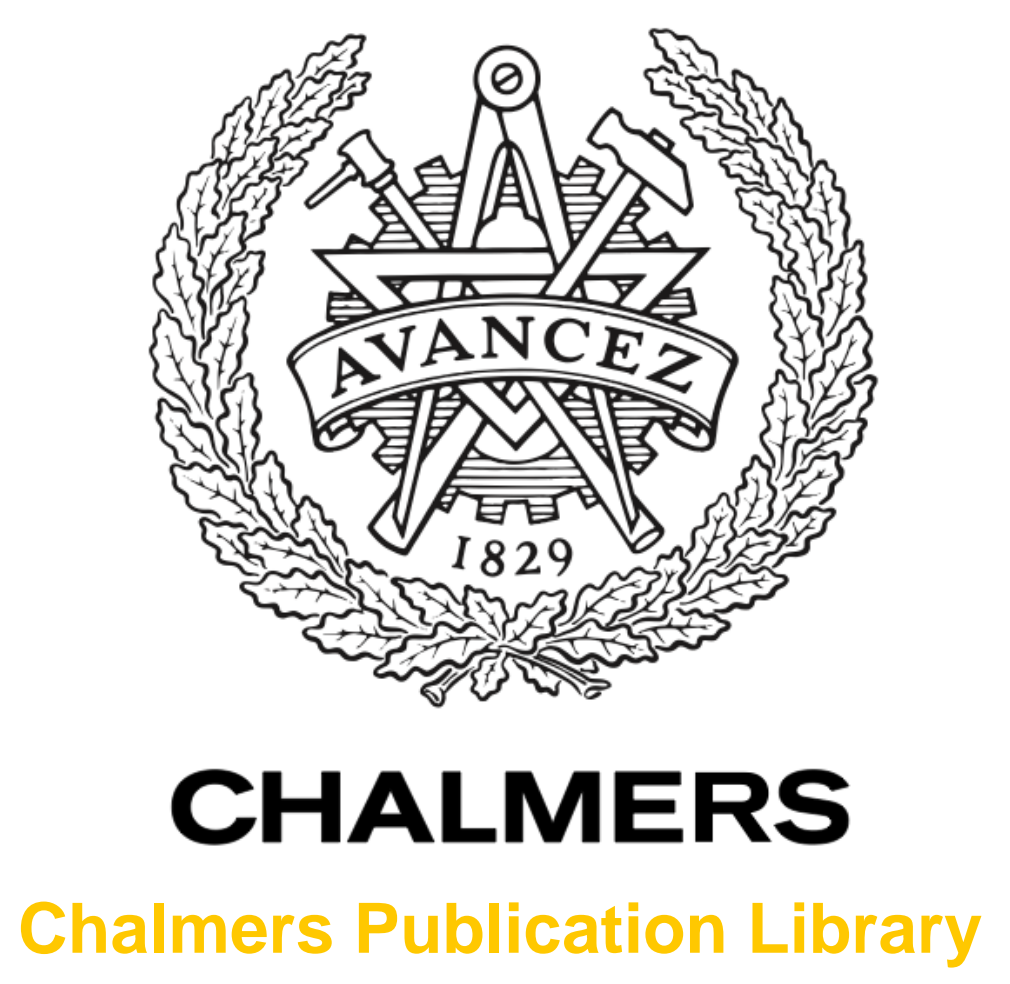

Satellite constellations: Towards the nonlinear channel capacity

This document has been downloaded from Chalmers Publication Library (CPL). It is the author's version of a work that was accepted for publication in:

25th IEEE Photonics Conference, IPC 2012, Burlingame, 23 - 27 September 2012 [Invited]

Citation for the published paper:

Agrell, E. ; Karlsson, M. (2012) "Satellite constellations: Towards the nonlinear channel capacity". 25th IEEE Photonics Conference, IPC 2012, Burlingame, 23 - 27 September 2012 [Invited] pp. 316-317.

http://dx.doi.org/10.1109/IPCon.2012.6358619

Downloaded from: http://publications.lib.chalmers.se/publication/170232

Notice: Changes introduced as a result of publishing processes such as copy-editing and formatting may not be reflected in this document. For a definitive version of this work, please refer to the published source. Please note that access to the published version might require a subscription.

Chalmers Publication Library (CPL) offers the possibility of retrieving research publications produced at Chalmers University of Technology. It covers all types of publications: articles, dissertations, licentiate theses, masters theses, conference papers, reports etc. Since 2006 it is the official tool for Chalmers official publication statistics. To ensure that Chalmers research results are disseminated as widely as possible, an Open Access Policy has been adopted.

The CPL service is administrated and maintained by Chalmers Library. 


\title{
Satellite Constellations: Towards the Nonlinear Channel Capacity
}

\author{
Erik Agrell and Magnus Karlsson \\ Chalmers University of Technology, Sweden \\ agrell@chalmers.se, magnus.karlsson@chalmers.se
}

(Invited)

\begin{abstract}
We present a family of adaptive constellations that, in a nonlinear optical fiber channel model, has a mutual information (modulation-constrained capacity) that does not decrease with signal power.
\end{abstract}

\section{INTRODUCTION}

The fiber nonlinearity is generally regarded as the most significant obstacle to increase the data rates in high-capacity fiber-optic systems [1]. Simulations and experiments show that the symbol- and bit-error rates (SER/BER) have local minima as functions of the average signal power for any given modulation format, in almost any investigated optical link [1]-[3]. It is then often concluded that that channel capacity (interpreted as the maximum achievable data rate for the given channel) will have a maximum at a certain signal power, beyond which the capacity will decrease. However, this is not correct in general, as was recently shown [4]. In contrast, the channel capacity is monotonically nondecreasing with signal power for all linear and nonlinear single-user channels. The reason for this apparent contradiction is that the channel capacity is defined as the maximum over all possible modulation formats and distributions, and such a search is generally infeasible with reasonable computational resources. For some specific nonlinear channels, the capacityincreases-with-power theorem has been shown [5]-[7], and the monotonically decreasing SER was illustrated in [8].

The purpose of this paper is to illustrate that no sophisticated optimization procedure is needed to obtain a family of modulation formats that perform well at arbitrarily high average power. We introduce a simple class of constellations, referred to as satellite constellations, whose performance improves, or stays constant, as the signal power increases. This class of constellations may have limited practical relevance, but, as we shall see, they offer a convenient technique to lowerbound the channel capacity (and upperbound the achievable SER). Moreover, the nonlinear regime is relatively unexplored and it it would be of general interest to find capacity-achieving constellations in this regime. The present study could be one small step towards this ultimate goal.

As a case study, we consider a simple kind of satellite constellations, based on phase-shift keying (PSK) modulation, and evaluate its performance over a nonlinear phase noise channel [9, Ch. 6], which accounts for inline amplifier noise and self-phase modulation, assuming negligible dispersion and cross-channel effects.

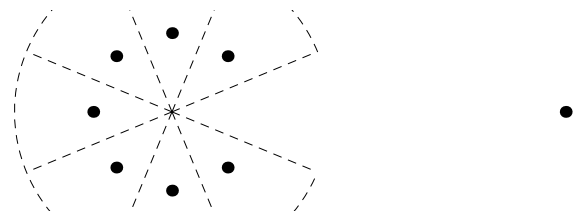

Fig. 1. A satellite constellation is characterized by a small probability mass having a much larger power than the rest of the constellation. This example shows a $(7,1)$-PSK constellation with $\beta=5$. Dashed lines indicate (suboptimal) decision regions.

\section{SATEllite Constellations}

We define a satellite constellation loosely as a constellation that has a high power in a small probability portion of the constellation. Intuitively, this means that most of the time, signal points are transmitted with moderate power, for which the channel is well-behaved.

Satellite constellations can be designed in many different ways. In this study, we modify a regular $M$-PSK constellation with amplitude $a$ into a satellite constellation by increasing the amplitude of just one of the $M$ points, the "satellite," to $\beta a$ for some parameter $\beta \geq 1$. While such a lone point may contribute little to capacity, it can consume arbitrarily high signal powers if located at a sufficiently high amplitude. The constellation, which we call $(M-1,1)$-PSK, is illustrated in Fig. 1.

An extreme case of a satellite constellation is nonuniform on-off keying, for which the power is zero with high probability. Such constellations, or variations thereof, have been used for certain channels to optimize the SER [10] or mutual information (MI) [6].

\section{Channel MOdel AND DETECTION}

As in previous works [2], [7], [8], [11], we use Ho's discrete-time model $[9, \mathrm{pp} .157,225-226]$. This model is valid in the limit of low accumulated local dispersion, e.g., for sufficiently low bandwidth or frequent inline compensation. The signal gets its main distortion from inline amplifier noise, which is additive, white, and Gaussian, together with a nonlinear self-phase modulation, which rotates the phase of the signal depending on its amplitude. The model was recently extended to dual polarizations in [3], but here we use the single-polarization version described in [2], with the same parameters: A $5000 \mathrm{~km}$ system is considered with a single wavelength, bandwidth (equal to the symbol rate) 42.7 $\mathrm{GHz}$, and distributed amplification. The fiber loss coefficient is $\alpha=0.058 \mathrm{~km}^{-1}$ (corresponding to $0.25 \mathrm{~dB} / \mathrm{km}$ ), the nonlinear coefficient is $\gamma=1.2(\mathrm{~W} \cdot \mathrm{km})^{-1}$, the amplifier 
noise parameter is $n_{s p}=1.41$, and the photon energy is $h \nu=1.28 \cdot 10^{-19} \mathrm{~W} / \mathrm{Hz}$. These parameters give a total optical noise power in the receiver of $-23.5 \mathrm{dBm}$, independently of the signal power. In the receiver, we apply the Lau-Kahn backrotation technique [2] to compensate for the deterministic part of the nonlinear phase noise, but due to the inline noise, a residual phase noise remains.

The detection rule is a regular $M$-PSK minimum-distance detector, except that a received signal with an amplitude above a certain threshold is always interpreted as the satellite, because when this point is transmitted, the system will experience a dramatically increased phase noise. This rule is suboptimal, but simple and sufficient for our purposes. In this study, the threshold is set somewhat arbitrarily to $2 a$. The decision regions are shown in Fig. 1.

\section{NUMERICAL RESULTS}

In Fig. 2, the SER is shown as a function of signal power $P$ for $(7,1)$-PSK satellite constellations with $\beta$ ranging from 1 (corresponding to regular 8-PSK with slightly suboptimal detection) to 10 . The minimum SER that can be attained does not depend appreciably on $\beta$, but the power at which this peak occurs grows approximately proportionally to $1+\left(\beta^{2}-1\right) / M$. Most importantly, even though the SER of any given $(M-1,1)$-PSK constellation displays a minimum at a certain power, after which it increases towards almost 1 , the minimum over all $\beta$ values does not increase. This envelope (dashed) serves as a simple upper bound on the achievable SER with adaptive modulation. The BER (not shown) displays essentially the same behavior. Stronger upper bounds can be obtained by optimizing the constellation, detection method, and thresholds. For instance, pure amplitude-shift keying is attractive in the nonlinear regime [2], [8].

The MI, which is shown in Fig. 3, also has a peak for any given $(M-1,1)$-PSK constellation, and the envelope of all such constellations (dashed) has not. Recalling that the channel capacity by definition is the supremum of the MI over all possible source distributions with a certain power, the envelope serves as a lower bound on channel capacity, a bound that can be strengthened if a wider class of source distributions is considered (including varying symbol probabilities or continuous distributions). This proves that the channel capacity does not decrease to zero, which is already known for this particular channel [7]. However, the proof method based on satellite constellations is applicable to any channel model [4].

\section{CONCLUSIONS}

We introduced satellite constellations as a means to transmit at an arbitrarily high signal power without losing performance, in terms of SER or MI, compared with standard modulation formats. The key idea is to rescale a portion of the constellations as the signal power increases, rather than the whole constellation linearly. This principle, which was here illustrated for a simple dispersion-free channel model, is applicable to arbitrary single-user channels. It provides theoretical insight into the design of capacity-achieving constellations in the nonlinear regime and may influence the design of adaptive modulation formats for nonlinear optical channels.

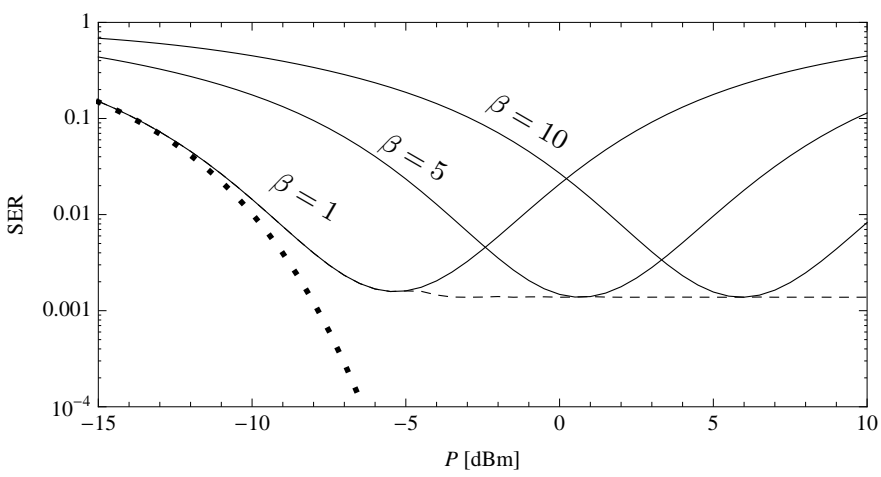

Fig. 2. Symbol error rates vs. signal power of $(7,1)$-PSK at a radius ratio of $\beta=1,5,10$ (solid), where $\beta=1$ corresponds to regular 8-PSK, and the minimum over all $(7,1)$-PSK constellations (dashed). The SER of 8-PSK for the linear Gaussian channel $(\gamma=0)$ is included for reference (thick dotted).

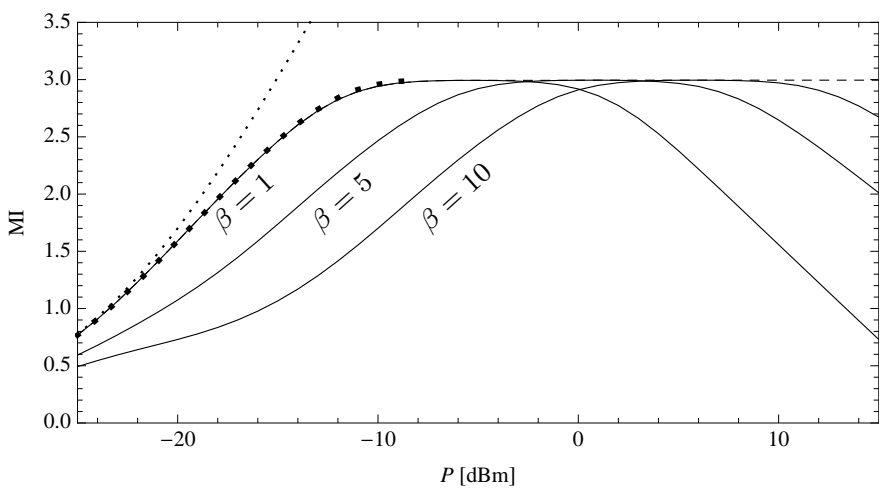

Fig. 3. MI of (7,1)-PSK constellations. The channel capacity is lowerbounded by the envelope of these curves (dashed line). Included for reference are the channel capacity (thin dotted) and the 8-PSK MI (thick dotted), both for the linear Gaussian channel.

\section{REFERENCES}

[1] R.-J. Essiambre, G. Kramer, P. J. Winzer, G. J. Foschini, and B. Goebel, "Capacity limits of optical fiber networks," J. Lightw. Technol., vol. 28, no. 4, pp. 662-701, 2010.

[2] A. P. T. Lau and J. M. Kahn, "Signal design and detection in presence of nonlinear phase noise," J. Lightw. Technol., vol. 25, no. 10, pp. 30083016, 2007.

[3] L. Beygi, E. Agrell, M. Karlsson, and P. Johannisson, "Signal statistics in fiber-optical channels with polarization multiplexing and self-phase modulation," J. Lightw. Technol., vol. 29, no. 16, pp. 2379-2386, 2011.

[4] E. Agrell, "The channel capacity increases with power," 2011, preprint. [Online]. Available: http://arxiv.org/abs/1108.0391

[5] K. S. Turitsyn, S. A. Derevyanko, I. V. Yurkevich, and S. K. Turitsyn, "Information capacity of optical fiber channels with zero average dispersion," Physical Review Letters, vol. 91, no. 20, p. 203901, 2003.

[6] K.-P. Ho, "Exact evaluation of the capacity for intensity-modulated direct-detection channels with optical amplifier noises," IEEE Photon. Technol. Lett., vol. 17, no. 4, pp. 858-860, Apr. 2005.

[7] M. I. Yousefi and F. R. Kschischang, "On the per-sample capacity of nondispersive optical fibers," IEEE Trans. Inf. Theory, vol. 57, no. 11, pp. 7522-7541, Nov. 2011.

[8] C. Häger, A. Graell i Amat, A. Alvarado, and E. Agrell, "Constellation optimization for coherent optical channels distorted by nonlinear phase noise," in Proc. GlobeCom, Anaheim, CA, Dec. 2012, to appear.

[9] K.-P. Ho, Phase-Modulated Optical Communication Systems. New York, NY: Springer, 2005.

[10] M. Steiner, "The strong simplex conjecture is false," IEEE Trans. Inf. Theory, vol. 40, no. 3, pp. 721-731, 1994.

[11] L. Beygi, E. Agrell, and M. Karlsson, "On the optimization of 16-point ring constellations in the presence of nonlinear phase noise," in Proc. Opt. Commun. Conf., Los Angeles, CA, Mar. 2011. 